Bibliotecas. Vol 38, № 2, julio - diciembre, 2020. EISSN: 1659-3286

URL: http://www.revistas.una.ac.cr/index.php/bibliotecas/index

DOI: $\mathrm{http://dx.doi.org/10.15359/rb.38-2.3}$

Licencia: Creative Commons (BY-NC-SA) 4.0 Internacional

\title{
Nuevos derroteros en el estudio de la felicidad: el análisis experimental de los tuiteros en México
}

\author{
New Paths in the Study of Happiness: An Experimental Analysis of Mexican Twitter \\ Users
}

Alejandro Ramos Chávez

Universidad Nacional Autónoma de México

Recibido: 28 de octubre de 2019

Aceptado: 12 de junio de 2020

Publicado: 30 de julio de 2020

\section{Resumen}

En la actualidad, es cada vez más común encontrarnos con formas alternativas para medir el desarrollo y bienestar de las naciones. Una de esas formas que ha cobrado reciente interés en círculos especializados de análisis y toma de decisión, es el de las mediciones de la felicidad de los pueblos. Por otro lado, el significante aumento en el uso de herramientas tecnológicas, como las redes sociales digitales, en aspectos como la obtención de información, la comunicación social, los intercambios de opiniones sobre los asuntos públicos y la generación en general de opinión pública, hacen que estas herramientas sean muy útiles para el entendimiento de múltiples fenómenos sociales. En este contexto, en México se han llevado a cabo esfuerzos a manera de análisis experimental que intentan conocer el estado de ánimo de la población utilizando como fuente el uso de las redes sociales digitales, de forma específica Twitter, mediante la utilización de los metadatos de esa red social. El presente trabajo pretende realizar un acercamiento teórico a los estudios de la felicidad, para posteriormente dar cuenta del caso específico de la medición de la felicidad de los tuiteros en México. Las conclusiones apuntan a destacar la viabilidad y el futuro promisorio de las mediciones experimentales, en este caso del manejo de los metadatos, para el análisis de diversos aspectos de la fenomenología social.

Palabras clave: Información, metadatos, felicidad, Twitter, México.

\section{Abstract}

It is increasingly common to find alternative ways to measure a country's development and wellbeing. One of these tools which has garnered increasing interest in specialized analysis and decision-making groups is measuring a population's happiness. Meanwhile, the 
Bibliotecas. Vol. 38, № 2, julio - diciembre, 2020. EISSN: 1659-3286

URL: http://www.revistas.una.ac.cr/index.php/bibliotecas/index

significant increase in digital tools for various uses, such as social networks, information gathering, social communication, opinion exchanges about public affairs and generation of public opinion, mean that these tools are very useful to understand multiple social phenomena. In this context, experimental analysis has been carried out in Mexico to try and understand the population's emotional state using social networks as a source, and specifically, Twitter, using Twitter metadata. This article aims to provide a theoretical approximation of happiness studies to subsequently use in the specific case of measuring happiness in Twitter users in Mexico. The conclusions aim to highlight the viability and promising future of experimental measurements, in this case metadata, to analyses various aspects of social phenomena.

Keywords: Information, metadata, happiness, Twitter, Mexico.

\section{Introducción}

A partir del surgimiento de conceptos como el de la era de la información y la sociedad del conocimiento, parecen haber quedado superadas las visiones que medían el desarrollo de las naciones a partir de aspectos vinculados con exclusividad a la riqueza y a la producción de los países. En este sentido, en la actualidad el desarrollo también puede ser medido tanto por el grado de conocimientos de la población, así como por otros aspectos que anteriormente quedaban en un plano subjetivo para estos análisis. Nos referimos de forma específica a la felicidad, que ha cobrado un interés amplio en su entendimiento como índice de bienestar de los países.

Por otro lado, el exponencial apropiamiento social de las tecnologías de la información y comunicación (TIC), también ha ocasionado que cada vez más investigaciones las analicen como fuentes importantes que permiten conocer diversos aspectos de la vida social. En este sentido, aspectos que hasta hace relativamente poco tiempo solo tenían parámetro de análisis en un entendimiento "físico" o "real" de los acontecimientos, en la actualidad tienen un importante análisis en la forma "virtual" o "en línea" de estos. Cada vez es más común escuchar aspectos como el de la cultura digital, la democracia digital, el gobierno digital y la ciudadanía digital, entre otros. De forma específica, este artículo se inscribe en las discusiones de la ciudadanía digital, pues se pretende dar cuenta de la utilización social que se le da a las TIC. Sin embargo, se hace referencia a la vinculación existente entre este tipo 
de ciudadanía con el gobierno, así como con las mediciones gubernamentales que de cierta forma se intentan llevar a cabo para conocer el funcionamiento y comportamiento social que se está generando a partir de la utilización de las redes sociales digitales.

En un primer momento la ciudadanía digital puede ser definida como un tipo de individuo que se caracteriza por la utilización de las TIC para participar, ya sea esporádica o frecuentemente, en la discusión e intercambio de información relacionada con las formas en las que están siendo tratadas las problemáticas sociales y encauzados los asuntos públicos. Esta participación e intercambios también pueden generar procesos de vinculación e injerencia con el gobierno, relativos al enfoque de la gobernanza, para plantear también alternativas para el tratamiento de los asuntos y problemas de interés social.

El trabajo está dividido en tres apartados generales. En el primero de ellos, se abordan algunos enfoques conceptuales de la temática de la ciudadanía, con mayor detenimiento al tipo de ciudadanía que interesa de forma particular en este trabajo, es decir el de la ciudadanía digital. En el segundo apartado, se analizan algunas de las tendencias académicas en el estudio de los estados de ánimo, desde una perspectiva social. De forma puntual se analizan dos enfoques que se consideran pilares en este contexto, el de la antropología de las emociones y el de la economía de la felicidad. En el tercer apartado, se toma en consideración el caso específico de un estudio experimental que lleva a cabo el Instituto Nacional de Estadística y Geografía (INEGI) de México, para conocer el estado de ánimo de los tuiteros en ese país. Por último, se concluye con algunas reflexiones finales, mencionando algunos derroteros que se vislumbra seguirán estos temas.

\section{De las plazas públicas a las pantallas: ciudadanía y ciudadanía digital}

Conviene en un primer momento definir la idea general de ciudadanía, con objeto de posteriormente entender al concepto específico de ciudadanía digital. En este sentido, la ciudadanía, desde una visión conservadora y atributiva al individuo, se entiende en este trabajo, como aquella a la que el gobierno le concede una serie de derechos y obligaciones, entre los que pueden destacar, relativo a los derechos, la elección de representantes públicos, 
de asociación, de acceso a la información tanto general como específicamente pública, así como los referentes a la libre manifestación de ideas. Por otro lado, desde un punto de vista más participativo, se entiende ciudadano como aquel individuo que constantemente interviene en el cauce de los asuntos públicos, es decir, como un individuo que no únicamente es destinatario de derechos y obligaciones, sino que también está interesado en participar, mediante la argumentación y la deliberación de ideas, en el rumbo que adquieren tanto las políticas como los asuntos públicos.

En este contexto, algunos autores (García-Canclini 1995; Lechner 2000; Winocur 2003a, 2003b) han analizado el tema de la ciudadanía no desde la visión predominante de la igualdad que conlleva el propio concepto, sino desde un enfoque de "ciudadanías diferenciadas", relacionadas con la búsqueda de reconocimiento como ciudadanos por parte de algunos grupos sociales, tomando en consideración sus particularidades o diferencias específicas. Lo que buscan estos grupos, como lo son los indígenas, las mujeres, los jóvenes, los adultos mayores, entre otros, es el reconocimiento de la existencia de diferentes identidades y problemáticas específicas de cada uno de ellos, mismas características que los hace acercarse o alejarse a los principios de igualdad incluidos en la idea general en la que se enmarca la ciudadanía.

Ahora bien, por ciudadanía digital podemos entender un tipo de ciudadano que utiliza las herramientas tecnológicas, sobre todo internet y las redes sociales digitales, para llevar a cabo sus procesos ciudadanos. Es decir, desde hacer valer sus derechos y cumplir sus obligaciones ciudadanas (pago de servicios gubernamentales, impuestos, etc.), así como para informarse sobre los asuntos públicos y participar en comunidades virtuales que, partiendo del mundo en línea, puede activar procesos y acción colectiva fuera de las pantallas. De esto último se desprende la idea ampliamente compartida de que la ciudadanía digital enmarca al individuo "capaz de ejercer su ciudadanía bajo un panorama de interactividad ofrecido por una plataforma digital" (Arcila, 2006, p. 18).

En la actualidad, es cada vez más común el uso social de aplicaciones y herramientas digitales como Wikis, Facebook, Twitter, Instagram y YouTube, usadas principalmente como 
fuentes que les permiten a los individuos obtener, generar, intercambiar y enriquecer información, pero también abren nuevas ventanas que permiten ventilar los asuntos públicos, llevar a cabo análisis políticos y sociales y generar opinión pública, pues de cierta forma tienen rasgos y características compatibles con aspectos del mundo no "virtual", como lo pueden ser: lugares de acceso a la información, encuentro social, debate, generación de opinión pública y espacios de presión política que en determinadas ocasiones puede desatar la acción colectiva. En este último punto, es interesante mencionar que, aunque no todas las actividades realizadas en el mundo virtual generen acción colectiva "real" o física", sí pueden llegar a generar cambios sociales y políticos. En este sentido, la viralización de información pública y de noticias políticas, puede llegar a hacer insostenibles los casos viralizados, por lo que los gobiernos modifican los procesos o castigan a las personas involucradas en esos casos, generando con ello un cambio.

Para el caso de México, existen algunos estudios que dan muestra de la importancia que están teniendo las TIC como instrumento social por parte de la ciudadanía digital. Ejemplo de esto es el Estudio sobre los hábitos de los usuarios de internet en México, del año 2019, con una muestra de 1968 entrevistas. En él que se menciona que los usuarios de internet en México han crecido de 20.2 millones de usuarios en el año 2006, a 82.7 millones de usuarios en el 2018 (Asociación de Internet.mx 2019). Otro dato interesante es que los usuarios utilizan internet en promedio ocho horas y veinte minutos diarios, dejando ya muy alejadas actividades que antes ocupaban gran parte del tiempo del ocio de las personas como ver la televisión (con dos horas veinte minutos) o escuchar la radio (con dos horas) ${ }^{1}$. Dentro de las actividades que más llevan a cabo los individuos en internet está la revisión de redes sociales digitales con un $82 \%$ de menciones, enviar o recibir mensajes con un $78 \%$, enviar o recibir mails con un $77 \%$ y la búsqueda de información con un $76 \%$. Estos datos resultan ilustrativos sobre la importancia que adquiere en la actualidad la utilización e intercambio de información a través de las redes sociales.

${ }^{1}$ Es oportuno tomar en consideración que actividades como ver películas, programas o series, así como escuchar la radio, también han transitado a un uso de aplicaciones digitales como Netflix, YouTube o Spotify, entre muchos otros. En este sentido, los datos de "ver la televisión" o "escuchar la radio", aquí presentados, toman en consideración a esas actividades sin la mediación de Internet. 
Por otro lado, un tema interesante desarrollado en este mismo estudio, pero en su versión del año 2018, fue el del internet y la democracia ${ }^{2}$. En este sentido 9 de cada 10 entrevistados consideraron que internet cambió sus hábitos para interactuar con el gobierno de México. De igual forma 6 de cada 10 entrevistados mencionaron que se han modificado sus formas de entender la democracia a partir del uso de internet, siendo principalmente la búsqueda de información sobre procesos electorales, la principal actividad llevada a cabo, con un $92 \%$ de incidencia.

Los principales lugares para la consulta y la difusión de información son las redes sociales (97\%), los sitios de noticias (79\%), los buscadores (74\%), además de videos/podcasts; blogs, foros y publicidad en línea con menor porcentaje (Asociación de Internet.mx 2018). Las problemáticas relacionadas con las barreras de acceso al internet siguen siendo: 1) la relativa poca oferta del servicio en algunas zonas del país, 2) los costos aún muy elevados de los servicios, y 3) la falta de capacidades individuales para usar las tecnologías.

Con este tipo de análisis, nos damos cuenta de los esfuerzos, a manera experimental, para conocer y medir los usos políticos, democráticos y participativos que lleva a cabo la ciudadanía mediante la utilización de internet y específicamente de las redes sociales digitales. En este sentido, en este trabajo interesan, de forma particular, los estudios de la felicidad, las mediciones de este tema y su vínculo con la ciudadanía digital.

Estos elementos dan paso al segundo de los temas del trabajo, pues desde hace algunos años se ha intentado medir el bienestar y desarrollo de los pueblos y las naciones no solamente con base en datos productivos, económicos y financieros, sino con perspectivas que apuntan más a cuestiones intangibles, como lo son los estados de ánimo. En este sentido, si bien existen críticas al mencionar que estos estudios son incompletos y subjetivos, han surgido análisis empíricos muy interesantes que tienen en cuenta la felicidad, con diversos indicadores, como componentes valiosos para medir el bienestar, por lo que convendría detenerse a considerar algunos de los elementos que dieron pie a un entendimiento teórico de estos estudios.

${ }^{2}$ Cabe destacar que en el año 2018 fueron de interés particular estos temas en México, pues se celebraron elecciones presidenciales, entre otros puestos de representación popular, por lo que fue particularmente útil conocer cómo los individuos utilizaban internet para vincularse con aspectos democráticos y de gobierno. 
Bibliotecas. Vol. 38, № 2, julio - diciembre, 2020. EISSN: 1659-3286

URL: http://www.revistas.una.ac.cr/index.php/bibliotecas/index

\section{El estado de ánimo como objeto de análisis social}

En un primer momento, resulta oportuno destacar de forma particular a dos teorías que han estudiado de alguna forma los estados de ánimo en función de su vinculación social. Estas teorías son la antropología de las emociones y la economía de la felicidad.

En la primera de estas teorías, es decir, en la antropología de las emociones, existen aportes muy interesantes que han analizado el estado de ánimo en el desarrollo o atraso de diversas comunidades, un referente en este sentido es el llevado a cabo por Frida Jacobo (2013), en su análisis de la situación de los nahuas de Cuetzalan, en el estado de Puebla, México; mismo análisis que brinda un abanico muy amplio y detallado del desarrollo teórico de esta línea de investigación de la antropología de las emociones. En este sentido, en un primer momento se puede hacer referencia al trabajo de Evans-Pritchard (1937) en el que se describe un comportamiento emocional generalizado entre los grupos africanos de los Azande ante aspectos naturales o sociales compartidos. Desde esta perspectiva, autores como Solomon (1984, p.4) han ido más allá al sostener que las emociones pueden estar construidas más por la cultura que por cuestiones biológicas, al sostener que:

las emociones deben interpretarse como adquisiciones culturales, determinadas por las circunstancias y conceptos de una cultura particular, mucho más que por las funciones de la biología y, más específicamente, de la neurología. Puede haber emociones universales, pero este es un asunto que debe resolverse empíricamente, no mediante un pronunciamiento a priori (Solomon 1984, p. 4).

Bajo estas perspectivas, siguiendo con los aportes de Jacobo (2013) una emoción, más allá de un sentimiento individual, también puede ser analizada como una construcción social, si partimos de que puede ser compartida mediante el lenguaje. Por lo tanto, a pesar de que los sentimientos pueden ser los mismos (esto puede ser sentimientos como el amor, miedo, ira, felicidad, etc.) existen variaciones en aspectos históricos y transculturales que los desatan o propician, por lo cual pueden ser analizados, según Lutz (1986) como aspectos construidos e incrustados como categorías sociales. En este punto convendría también abordar el estudio del concepto de embeddedness o incrustación, de Granovetter (1985), desde esta perspectiva 
de la economía a las estructuras sociales. En este sentido, se menciona la conexión entre las personas, más que por un contexto compartido, por diferentes cuestiones de integración imbricadas, como lo son las emociones que dan soporte y sustento al plano social.

En términos generales la antropología de las emociones considera que las emociones se encuentran fuertemente establecidas en el ámbito estructural y funcional de lo social, pues su eje rector es visualizado en la cultura, lo anterior por principalmente tres aspectos:

1) las emociones cumplen una función tanto a nivel individual como a nivel social, pues poseen contenidos ideológicos que se transmiten culturalmente;

2) las emociones pueden ser situadas dentro de un contexto histórico y social determinado;

3) las emociones forman parte de la estructura social, pues vinculan al individuo con su sociedad al mismo tiempo que la construyen.

Por otro lado, en relación con la "economía de las emociones", se puede destacar un antecedente muy importante en el libro Los felices 90. La semilla de la destrucción (2003), de Joseph Stiglitz. En este libro, Stiglitz vislumbra un principio de siglo muy turbulento debido a los acontecimientos de la aparente estabilidad política, económica y financiera de la última década del siglo pasado en Estados Unidos de América. Ante esta situación Stiglitz menciona que no solo sería ese país el único que sufriría los embates de las crisis, sino que serían todos los países que dependen económicamente de él. Por lo tanto, podemos ubicar el análisis de la supuesta felicidad, precisamente en la estabilidad de la última década del siglo pasado, pero que a la postre, sería el principio de las fuertes crisis venideras. Para intentar definir este concepto, siguiendo las propuestas de Stordeur, se puede mencionar que:

La economía de la felicidad consiste en una rama de la economía de la conducta que desafía también algunas de las asunciones centrales de la economía tradicional y busca, básicamente por medio del empleo de reportes de utilidad obtenidos en encuestas, recabar información sobre las preferencias de las personas de una manera alternativa a la típica de la economía tradicional. Este enfoque se basa, entonces, en encuestas donde las personas reportan su nivel de felicidad en una escala finita para luego emplear herramientas estadísticas con la finalidad de establecer correlaciones entre diversas variables y niveles de felicidad" (2014, p. 86). 
Un elemento que desató el interés por medir el bienestar desde otros enfoques consistió en los cuestionamientos más serios que se les hizo a los economistas tradicionales, quienes veían relación directa, y casi exclusiva, entre el nivel de ingreso y el bienestar, pues un gran número de estudios demostraron que las personas podían ser cada vez más ricas, pero no necesariamente más felices. Bajo estas perspectivas podemos destacar los aportes de Amartya Sen, quien en parte de su obra se interesó por estudiar no solo la disponibilidad de satisfactores, sino las capacidades de poderles sacar provecho, que nos llevarían a las realizaciones de satisfacción. Sen (2000), tomando en consideración estas perspectivas, se aleja de la visión dominante en la economía de libertad negativa (relacionada con el no impedimento de acción) y avanza hacia la libertad positiva, es decir, las capacidades reales de un individuo de ser o hacer algo.

Estos planteamientos han sido tomados en consideración, cada vez con mayor importancia, por organismos internacionales para medir el grado de bienestar de las naciones. Ejemplo específico de esto son los estudios llevados a cabo por la Red para el Desarrollo Sostenible de las Naciones Unidas, quien desde el año 2012, lleva a cabo un estudio denominado "Reporte de la felicidad" o "World Happiness Report" cuya versión del año 2018 (Helliwell, Layard, y Sachs 2018) comparó a 156 naciones, tomando en consideración seis variables específicas: ingreso, expectativa de vida saludable, apoyo social, confianza, libertad y generosidad.

En los resultados de este último estudio, y teniendo en cuenta que México resulta un país muy mal calificado en cuanto a la percepción de corrupción ${ }^{3}$, además de los problemas de pobreza, inseguridad y violencia, se ubica en sitio 24 de los 156 países tomados en consideración. Siendo con ello el segundo país más feliz de América Latina, pues apenas es superado por Costa Rica, quien ocupa la posición número 13. Según los especialistas, la felicidad de gran parte de los países de América Latina radica principalmente en la calidez y fortaleza de las relaciones familiares, las cuales son vistas en estos países como condicionantes para alcanzar la felicidad sobre otros aspectos económicos o políticos.

${ }^{3}$ Según el Índice de Percepción de Corrupción, llevado a cabo por Transparencia Internacional, México, en el reporte del año 2017, ocupa el lugar 135 de las 180 naciones analizadas (Transparency International 2018), mientras que en el 2010 se ubicaba en el lugar 98, en el 2000 en el 59 y en 1995 en el 32. Es decir, ha sido constante el incremento en la percepción de la corrupción en el país a lo largo de la realización de este estudio, lo que de cierta forma se contrapone con la idea general de la felicidad, en la cual México constantemente se encuentra en el primer cuartil de las posiciones. 
Existen estudios similares que, a manera experimental, están intentando medir la felicidad de las comunidades virtuales, tal es el caso del estudio del "estado de ánimo de los tuiteros en México", que serán abordados de forma general en el siguiente apartado del trabajo.

\section{El estudio sobre el "Estado de ánimo de los tuiteros en México"}

Desde el año 2015 el Instituto Nacional de Estadística y Geografía (INEGI) lleva a cabo el estudio del estado de ánimo de los tuiteros en el país. Lo anterior representó, según las instituciones participantes en este proyecto

un paso hacia el uso de fuentes alternativas de Big Data para generar nuevas estadísticas experimentales. Se logró concretar una aplicación, para el ámbito de Bienestar Subjetivo, en la que se presentaba el resultado de todo el ciclo necesario en el uso de técnicas de Big Data utilizando fuentes no tradicionales como lo son las redes sociales. Este ciclo abarca desde la recolección de la fuente de datos, el análisis de éstos, su preprocesamiento, la clasificación y el procesamiento de cada dato, su almacenamiento, la generación de indicadores, hasta la representación visual para difundir dichos resultados (Instituto Nacional de Estadística y Geografía 2017, p. 1).

En este análisis se incursiona en aplicaciones prácticas de Ciencia de Datos, entendida por William Cleveland (2007) como un campo que incluye: análisis de datos, modelos estadísticos, métodos de construcción de modelos, métodos de estimación para realizar inferencia estadística, sistemas de hardware y software, algoritmos computacionales, herramientas estadísticas, entre otras. Lo anterior, fue utilizado por el INEGI con objeto de brindar estudios de bienestar subjetivo, utilizando Twitter y fuentes de Big Data, para acercarse a determinar el estado de ánimo de los tuiteros del país.

En este contexto, en el documento metodológico del estudio se menciona que Twitter es una red social en donde los usuarios pueden escribir textos cortos de hasta 280 caracteres (originalmente 140), mismos textos que quedan visibles de forma pública. Los mensajes pueden ser georreferenciados para conocer la ubicación exacta desde donde sale el texto. Cabe señalar que para este estudio del estado de ánimo sólo se utilizan mensajes que tengan 
ese atributo para poder realizar los estudios geográficos, con objeto de no solo conocer el estado de ánimo general (del país) sino de cada una de las entidades federativas.

En este estudio, se siguió una metodología de machine learning (aprendizaje automático) muy interesante para generar la estadística del estado de ánimo de los tuiteros. En este sentido, en un primer momento se etiquetaron múltiples veces, con objeto de alcanzar un consenso en la etiqueta, 4000 tuis por más de 5000 estudiantes de la Universidad Tec Milenio. Posteriormente se llevó a cabo una limpieza y normalización de tuits para disminuir el desorden en las calificaciones. En este punto se eliminaron contradicciones y repeticiones y se identificaron los tuits con mayor consenso en su etiqueta. También se utilizó un proceso de normalización con objeto de corregir errores, anonimización de usuarios, aprovechamiento de emoticones e identificación de sintaxis. Con respecto a los emoticones se clasificaron 512 de uso popular en cuatro clases (positivo, negativo, neutro y ninguna) (Instituto Nacional de Estadística y Geografía 2017).

Posteriormente se llevaron otros procesos de definición de conjuntos de entrenamiento y validación, desarrollo y entrenamiento de clasificadores automáticos, clasificación masiva de tuits, además de un procesamiento para distinguir tuiteros locales de visitantes. Esto que se menciona enunciativamente representó para los desarrolladores retos específicos en cada etapa para generar la herramienta utilizada en la actualidad, para clasificar los tuits con carga positiva y aquellos con carga negativa.

Para aprovechar de mejor manera la aplicación, el programa no solo brinda las gráficas del estado de ánimo de los tuiteros del país por día, semana o mes, sino que las gráficas son acompañadas con datos muy interesantes para, de cierta forma, conocer o intuir el porqué del estado de ánimo generalizado en el país, en una región o en un estado determinado, así como la posibilidad de comparar el estado de ánimo de los tuiteros en dos entidades federativas distintas o entre una de ellas con el global nacional.

Ejemplo de los datos que también son puestos a disposición por el programa, son un gráfico sobre el número de tweets que son escritos por hora del día, ofreciendo una visualización de 
los horarios de mayor uso de esta red social en el país. De igual forma, es posible visualizar en una nube, realizada por día, los temas que son tendencia, mejor conocidos como trending topics, que se van formando a partir de las etiquetas más utilizadas o hashtags. Lo anterior da muestra del trabajo profundo de metadatos utilizados por estas estadísticas, con la intensión de identificar los temas del momento que pueden influir en el estado de ánimo de los usuarios de esta red social. Finalmente, otro recurso que brinda esta aplicación es el de conocer las noticias del día que pudieron influir en el sentimiento colectivo, por lo que la aplicación da la posibilidad de acceder a las noticias que acontecieron en un día determinado directamente el buscador de noticias de Google o por la página kiosko.net.

Sin lugar a duda, este estudio abre una perspectiva de análisis innovadora en cuanto al análisis de la situación de la población del país, pues los datos no solo se basan en aspectos económicos o censales, sino que toma en consideración la perspectiva de los sentimientos de la población, específicamente reflejada en la carga negativa o positiva en la que son escritos y son compartidos los Tweets. Lo anterior abre una perspectiva amplia tanto en el uso como en el estudio de los metadatos y su incidencia en el estudio de lo social.

\section{Reflexiones finales}

El concepto de ciudadanía ha transitado de un enfoque atributivo del conjunto de derechos y obligaciones concedidas por el Estado, a un tipo de ciudadanía entendida en un plano más participativo que se vincula no solo con la capacidad de emitir un voto en momentos coyunturales de elección de representantes públicos, sino con un abanico mucho más amplio de injerencia e involucramiento con los asuntos del orden de lo público-políticogubernamental. En este contexto, también se han multiplicado los estudios que abordan o intentan describir ya no una única forma de ciudadanía, sino de ciudadanías diferenciadas que se vinculan con las situaciones específicas en las cuales se encuentran los propios ciudadanos a estudiar. Esto es, dejar de lado el enfoque de ciudadanía universal y pasar a un enfoque mucho más centrado en las características económicas, sociales y culturales específicas de los grupos analizados. Ejemplos de estos grupos o ciudadanías diferenciadas pueden ser los adultos mayores, los jóvenes, las mujeres, los grupos indígenas, las personas 
con capacidades distintas y los conjuntos sociales con gustos y preferencias específicas. La ciudadanía específica que abordó este estudio es el de la ciudadanía digital, relacionada con aquella ciudadanía que utiliza los medios digitales tanto para hacer valer sus derechos y cumplir con sus obligaciones, como en el enfoque atributivo de la ciudadanía antes descrito, así como por el uso de estas mismas herramientas digitales para generar procesos informativos y participativos que intenten de alguna forma $u$ otra, modificar las estructuras sociales, políticas o culturales tanto de las localidades en las que se encuentran, así como de opinar y actuar ante los problemas y retos transnacionales.

Por otro lado, parecen superados los estudios que intentan medir la riqueza y bienestar de las naciones a partir, casi con exclusividad, desde perspectivas economicistas, productivistas y de medición de los ingresos, a formas más amplias de medir el bienestar de las naciones y grupos sociales. En este contexto surgen los modelos y estudios que miden el bienestar a partir de cuestiones subjetivas de análisis como lo son los estudios de las percepciones de la felicidad de las naciones. Estos estudios no se han quedado al margen de la situación que se da en los ambientes digitales, por lo que también han surgido estudios específicos que intentan medir estos mismos elementos, pero de la parte de la población que usa cotidianamente las herramientas digitales.

Tomando en consideración estos antecedentes, en este trabajo se abordó el estudio que, a manera de estudios experimentales, se está llevando a cabo sobre la felicidad de los tuiteros en México; en forma de estadística experimental intenta conocer cómo se encuentra el estado de ánimo de la población usuaria de redes sociales, específicamente de Twitter, y su vinculación con la información de los momentos en los que surgen las muestras. Lo anterior presupone un interesante camino de análisis del plano social a partir de la utilización de Big data.

Con estos trabajos se abre la amplia posibilidad del uso y análisis de metadatos, con objeto de identificar patrones que reflejen la situación o situaciones en las que se encuentren las poblaciones de una nación. En este trabajo interesó de forma particular el estado de ánimo reflejado en la utilización de las redes sociales, específicamente en Twitter, sin embargo, 
estos estudios pueden extenderse tanto a otras redes sociales, como a otros tópicos de análisis que pueden incluir minería de datos para el análisis político, de injerencia pública o de vinculación gubernamental con la ciudadanía para el tratamiento y cause de los asuntos públicos. De igual forma, resultaría interesante construir una tipología sobre los diferentes usuarios de estas redes sociales digitales, con objeto de conocer su comportamiento específico ante diversas situaciones de orden político, económico, social o cultural. Con este análisis se podría conocer el perfil de los usuarios de las diferentes redes sociales digitales, para conocer si algunas de ellas son más proclives de desatar procesos participativos o políticos que en otras. Estos elementos nos demuestran la viabilidad futura de estos temas de investigación para ser abordados por especialistas de muchas áreas de especialización, entre las que destacan la Bibliotecología y los Estudios de la Información.

Si bien estos temas quedan como derroteros de investigaciones futuras, van estableciendo en la actualidad nuevas formas de comportamiento que dan vida a las situaciones políticas, sociales, económicas y culturales de las naciones, pues el potencial de la información para el análisis y entendimiento de lo social que pueden generar los metadatos, la utilización y apropiación de las herramientas digitales por parte de la ciudadanía, así como la utilización de estas herramientas por parte del gobierno para llevar a cabo la gestión pública y el acercamiento con sus ciudadanos, son ya aspectos que forman parte integral del entendimiento de la situación de los pueblos y las naciones.

\section{Bibliografía}

Arcila, C. (2006). El ciudadano digital. Revista Latinoamericana de Comunicación CHASQUI, (93), 18-21.

Asociación de Internet.mx. (2018). Estudio sobre los hábitos de los usuarios de Internet en México 2018. Recuperado de http://www.asociaciondeinternet.org.mx/es/component/remository/Habitos-deInternet/lang,es-es/?!temid= 
Cleveland, W. (2007). Data Science: An Action Plan for Expanding the Technical Areas of the

Field of Statistics. International Statistical Review, 69 (1), 21-26. https://doi.org/10.1111/j.1751-5823.2001.tb00477.x

Evans-Pritchard, E. (1937). Witchcraft, Oracles and Magic among the Azande. Oxford: Oxford University Press.

García-Canclini, N. (1995). Consumidores y ciudadanos. Ciudad de México: Grijalbo.

Granovetter, M. (1985). Economic Action and Social Structure: The Problem of Embeddedness. American Journal of Sociology, 91, 481-510.

Helliwell, J; Layard, R. y Sachs, J. (2018). World Happiness Report 2018. Nueva York: Sustainable Development Solutions Network.

Instituto Nacional de Estadística y Geografía. (2017). Estado de ánimo de los tuiteros en los

Estados Unidos Mexicanos. Documento metodológico. Segunda edición. Recuperado de http://www.beta.inegi.org.mx/app/biblioteca/ficha.html?upc=702825099718

Jacobo-Herrera, F. (2013). Hacia una antropología de las emociones. La atención de la envidia entre los nahuas de Cuetzalan, Puebla. Doctorado, Ciudad de México: CIESAS.

Lechner, N. (2000). Nuevas Ciudadanías. Revista de estudios sociales, (5), 25-31.

Lutz, C. (1986). Emotion, Thought, and Estrangement: Emotion as a Cultural Category. Cultural Anthropology, 1 (3), 287-309.

Sen, A. (2000). Desarrollo y libertad. Buenos Aires: Planeta.

Solomon, R. (1984). Getting Angry: The Jamesian Theory of Emotion in Anthropology. En R. C. Solomon (ed.), Not Passion's Slave, (1-23). Recuperado de Oxford: Oxford University Press.

https://www.oxfordscholarship.com/view/10.1093/0195145496.001.0001/acprof9780195145496-chapter-6

Stiglitz, J. (2003). Los felices 90: La semilla de la destrucción. Barcelona: Taurus.

Stordeur, E. (2014). Felicidad, bienestar y políticas públicas: algunas consideraciones preliminares. Revista Teoría del Derecho de la Universidad de Palermo, 1 (1), 83-114.

Transparency International. (2018). Corruption Perceptions Index 2017. Nueva York.

Winocur, R. (2003aㅡ). Ciudadanos en los medios. La construcción de lo público en la radio. Buenos Aires: Gedisa. 
Winocur, R. (2003b). La invención mediática de la ciudadanía. En P. Ramírez (Ed.) Espacio público y reconstrucción de ciudadanía, (231-252). México D.F.: Miguel Ángel Porrúa.

\section{Datos de autoría}

Alejandro Ramos Chávez: Instituto de Investigaciones Bibliotecológicas y de la Información, Universidad Nacional Autónoma de México. Correo electrónico: aramos@iibi.unam.mx 